\title{
Les langues vivantes dans les établissements éducatifs russes au siècle des Lumières
}

En amont de l'histoire de l'enseignement du français aux russophones

\section{Nadejda Kouzmina}

\section{(2) OpenEdition}

\section{Journals}

Édition électronique

URL : https://journals.openedition.org/dhfles/1057

DOI : $10.4000 /$ dhfles. 1057

ISSN : 2221-4038

\section{Éditeur}

Société Internationale pour l'Histoire du Français Langue Étrangère ou Seconde

Édition imprimée

Date de publication : 1 décembre 2005

Pagination : 7-26

ISSN : 0992-7654

Référence électronique

Nadejda Kouzmina, « Les langues vivantes dans les établissements éducatifs russes au siècle des Lumières », Documents pour l'histoire du français langue étrangère ou seconde [En ligne], 35 | 2005, mis en ligne le 29 mai 2011, consulté le 27 mai 2021. URL : http://journals.openedition.org/dhfles/1057 ; DOI : https://doi.org/10.4000/dhfles.1057

Ce document a été généré automatiquement le 27 mai 2021.

(C) SIHFLES 


\section{Les langues vivantes dans les établissements éducatifs russes au siècle des Lumières}

En amont de l'histoire de l'enseignement du français aux russophones

\section{Nadejda Kouzmina}

1 Sous le règne de Catherine II (1762-1796), et pour la première fois dans l'histoire russe, furent jetés les fondements d'un système d'instruction et d'éducation à caractère général.

2 Dans son désir d'appliquer les idées des Lumières, en vue de transformer la société par la création et la reproduction d'une nouvelle race d'hommes, libérés des vices que développe la société, l'impératrice éclairée se fixa pour objectif d'organiser une instruction et une éducation exemplaires, d'abord pour la noblesse, l'élite de la société russe, et ensuite pour les autres couches sociales.

3 Peu après son accès au pouvoir, en 1762, elle confia l'élaboration du projet et des programmes pour les établissements éducatifs à Ivan Ivanovitch Betzky (1704-1795), un homme connu pour son érudition et sa connaissance des théories pédagogiques de l'Europe des Lumières. Pour réaliser cette tâche importante, Betzky s'inspira des idées de Locke, de Montaigne, de Rousseau ainsi que des expériences faites dans le domaine éducatif par plusieurs pays comme le Danemark et l'Autriche, mais également de l'héritage de l'Antiquité romaine et de la Perse, sans parler d'autres apports.

4 L'instruction et l'éducation adoptées dans les établissements déjà existants à SaintPétersbourg pour les jeunes nobles allaient être considérablement transformées. En effet, le Corps impérial des cadets nobles, fondé en 1731, et l'Académie des Beaux-arts, ouverte en 1745, obtinrent respectivement en 1766 et 1764 de nouveaux Statuts qui valorisaient à la fois des objectifs pédagogiques et le souci d'une instruction générale et spécialisée ${ }^{1}$.

5 En 1763, sur l'initiative de Betzky, fut crééeà Moscou la Maison des enfants trouvés, une institution destinée à accueillir des enfants abandonnés à la naissance par leur mère. 
Puis la Maison des enfants trouvés intégra rapidement la Maison de l'éducation. Dans ces établissements, lesenfants des milieux défavorisés pouvaient poursuivre leurs études jusqu'à dix-huit ans avant de devenir des sujets utiles à la société. Bien instruits, éduqués, maitrisant un métier, ils devaient, selon Betzky, constituer progressivement un tiers état pour la Russie de l'époque et pour celle du futur. L'ouverture de ces écoles accéléra l'apparition d'établissements analogues s'inspirant de ce modèle : une école de commerce à Moscou (1772), une nouvelle Maison de l'éducation (1772) à SaintPétersbourg, une école attachée auCorps des cadets, accueillant des garçons de condition modeste, qui suivaient cependant les mêmes programmes que les jeunes gens de la noblesse.

6 Catherine II jeta également les premières bases de l'éducation féminine en Russie en ordonnant la fondation à Saint-Pétersbourg de l'Institution des jeunes filles nobles et, un peu plus tard, celle des filles de la bourgeoisie. Ces institutions pour les filles, connues sousle nom d'Institutions Smolny, devaient ressembler à la célèbre maison de Saint-Cyr près de Paris, mais avec prédominance d'éléments d'éducation laïque².

\section{Le projet d'éducation de Betsky}

7 La publication du Projet général de l'éducation de la noblesse des deux sexes et des Statuts des différents établissements éducatifs rédigés par Betzky date de $1764^{3}$. Dans ces textes, Betzky développe ses principes pédagogiques essentiels. Parmi ses thèses principales on note l'interdiction absolue des châtiments corporels, une bonne hygiène et le souci du développement physique, l'acquisition progressive de nouvelles connaissances, l'éveil de la curiosité et du goût pour l'effort intellectuel, l'ambiance d'amitié mais aussi l'émulation entre les élèves, etc. On reconnaît là les principes défendus par des hommes éclairés tels que Fénelon, Rousseau, Locke, Milton et d'autres.

8 L'application de ces principes au sein des établissements éducatifs ne fut pas facile. Elle suscita bien des problèmes, dont le plus important fut le recrutement d'enseignants qualifiés qui fussent également partisans du mode d'éducation préconisé.

9 Nombre de contemporains, souvent proches de la philosophie des Lumières, s'intéressèrent à l'expérience russe, voulurent se tenir informés et manifestèrent à son sujet des opinions diverses, certaines très favorables ou admiratives, d'autres plus réservées ou plus critiques.

10 Les historiens russes divergent également à propos de ces établissements et de leur importance pour la formation de l'élite intellectuelle de la société russe du XVIII siècle et du début du XIX $\mathrm{X}^{\mathrm{e}}$ siècle. On leur a souvent reproché leur caractère élitiste, le côté superficiel de leur enseignement, la soumission aux modèles étrangers et surtout la mainmise des Français. On critiquait le rôle que jouaient les langues française et allemande tenues pour des matières fondamentales. L'attention portée aux langues vivantes dans ces établissements paraissait disproportionnée et nuisible pour la langue maternelle. L'idée que la noblesse russe préférait s'exprimer en françaisparce qu'elle avait une maîtrise insuffisante de l'idiome national s'implanta comme lieu commun durable dans les manuels d'histoire de la didactique des langues.

11 Ces idées reçues masquent la réalité. En fait, les talents des anciens élèves du Corps des cadets ou des établissements similaires de l'époque de Catherine II ne se limitaient pas à la maîtrise de la langue de Molière. Plusieurs d'entre eux devinrent des maitres de 
plume nationaux et se firent remarquer par leur œuvre littéraire en russe ainsi que par leurs traductions. Plus que par leur formation militaire, c'est bien souvent par leur érudition encyclopédique et par d'autres compétences que les anciens élèves du Corps des cadets de l'époque se signalèrent à l'attention de leurs contemporains ${ }^{4}$.

De plus, ces idées reçues n'ont guère stimulé l'intérêt des chercheurs pour les détails et pour les méthodes d'un enseignement aussi désapprouvé. Si l'on s'intéresse de près au contenu de l'enseignement du français, de l'allemand ou de la langue maternelle dans ces établissements, on risque de rester sur sa faim. Il existe bien un corpus de documents, d'outils didactiques relatifs à l'enseignement des langues dans ces établissements, mais il est resté jusqu'à présent inexploré. On connaît mal les enseignants de ces établissements, leurs conceptions et leurs parcours pédagogiques 5 .

Le but de cet article est, en s'appuyant sur les témoignages des acteurs, de soulever un coin du voile sur la nature de l'enseignement du français et des langues vivantes au sein de l'Institution du Corps impérial des cadets destiné aux enfants de la noblesse, ainsi que sur son histoire.

\section{L'Institution du Corps impérial des cadets}

14 Nicolas Gabriel Le Clerc (1726-1798), savant, médecin, écrivain français qui servit de nombreuses années la couronne russe et laissa plusieurs ouvrages sur la Russie deson temps compte parmi les fidèles collaborateurs de Betzky dont il avait fait la connaissance à Paris avant 1762. Il occupa le poste de directeur des études ${ }^{6}$ dans le Corps impérial des cadets entre 1769 et 1773 pendant la période où l'établissement était administré par Betzky. Deux ans plus tard, en 1775, Nicolas Le Clerc traduisit en français ${ }^{7}$ tous les projets et statuts rédigés par Betzky pour les établissements éducatifs russes. La traduction fut emportée et éditée à Amsterdam par les soins de Diderot qui tenait à informer l'Europe de l'initiative de Catherine II $^{8}$. Outre les textes de Betzky, l'ouvrage comporte plusieurs contributions importantes de la plume de Nicolas Le Clerc lui-même : une Introduction nécessaire à l'ouvrage par le traducteur de trente-deux pages, des Réflexions du traducteur sur l'éducation des demoiselles, etc.

L'Introduction est très élogieuse à l'égard de Catherine et de Betzky. Le Clerc défend avec force leurs vues sur les objectifs de l'instruction et de l'éducation de la jeunesse. Pour ses réformes grandioses, dit-il, "cette Princesse avait besoin d'un homme : elle le trouva.» (Introduction, p. 6)

16 Pour souligner l'importance de l'œuvre de Betzky, Le Clerc affirme qu'à cette époque « la Russie était la seule Nation de l'Europe, qui eût un système suivi d'éducation depuis l'âge de cinq ans jusqu'à vingt, système d'autant plus solide que, dans la pratique, on se rapproche, autant qu'il est possible, des lois de la Nature et des droits de la raison ».

17 Le Clerc explique ensuite pourquoi il a entrepris la traduction des œuvres de Betzky en français :

Nous naissons citoyens: nos rapports avec tous les hommes nous rendent cosmopolites. Voilà la profession de foi de l'honnête homme ; c'est la mienne. Je n'ai ni faveur à prétendre, ni disgrâce où retomber. L'intérêt, ou la flatterie, n'entrent donc pour rien dans les motifs qui m'ont engagé à rendre publiques ces Institutions. J'ai entrepris de mon chef un ouvrage plus pénible que glorieux pour le traducteur, parce que je l'ai jugé utile à toutes les nations. Quiconque aura lu ces Institutions, conviendra que chacune d'elles est un monument élevé à la sagesse de 
l'Impératrice. [...] Quelle sublime leçon le pouvoir absolu donne aujourd'hui au monde ! [...] et c'est le Nord qui présente cet exemple à l'Orient et au Midi. » (Ibid., p. 6-7)

\section{La place de l'enseignement des langues vivantes}

18 de l'enseignement des langues vivantes et anciennes dans l'éducation des cadets :

(T. 2, p. 56) : Institution du Corps des cadets :

De ce qui doit être enseigné aux élèves.

L'art de guerre étant l'objet principal de l'institution du Corps des cadets, doit faire [...] la base de leur éducation. [...] On joindra à ces exercices l'étude des sciences suivantes :

La langue esclavonne ${ }^{9}$ pour les mettre en état d'écrire correctement et élégamment en russe, et pour leur donner l'intelligence facile des livres canoniques et autres concernant leur patrie. On y joindra les langues étrangères et principalement la française, en les y exerçant continuellement par l'usage, pour les leur rendre familières. Cette méthode est préférable à une étude perpétuelle des règles de la grammaire. Il ne faudrait pas négliger cependant de leur en apprendre les principes, afin qu'ils pussent les connaître à fond; mais cette connaissance n'appartient qu'à la raison éclairée et réfléchissante [...].

Il n'importe pas moins à l'éducation des élèves, de leur apprendre la langue esclavonne, selon les règles de grammaire, et de composer à cet usage un vocabulaire esclavon-russe, et russe-esclavon, en y ajoutant, dans la même langue, des abrégés bien faits de tout ce qui l'on doit enseigner au Corps. (Ibid., p. 63)

On ne doit pas négliger ici une observation très importante ; c'est que, si les élèves n'apprennent la géographie, l'histoire et les autres sciences qu'en langue étrangère, ils en retireront peu d'utilité. A l'âge de quatorze à quinze ans, elles leur paraîtront tout à fait extraordinaires dans leur langue naturelle[maternelle]. Il en est de même des autres langues qu'ils n'apprendront jamais à parler correctement, s'ils ne se servent au Corps que de la langue russe; il faut donc les exercer également dans toutes. La preuve de ce que l'on avance ici est prise dans le Corps même. Les Livoniens et autres Allemands, qui étudient dans leur langue naturelle, font des progrès beaucoup plus grands et plus rapides que les Russes. Il est difficile à la vérité, peut-être même impossible de trouver actuellement assez de précepteurs pour enseigner les sciences en langue russe, et les étrangers savants ne l'entendent pas. Mais cette difficulté peut être facilement levée dans l'espace de cinq à six ans. De bons appointements et d'autres motifs d'encouragement engageront ces étrangers à s'instruire de notre langue, et nous aurons par là les ressources qui nous manquent. On a pour exemple celui de M. le professeur Euler, qui a enseigné à Berlin la géométrie en langue russe, à une de nos personnes qualifiées. (Ibid., p. 62)

Les Statuts du Corps révèlent l'importance de l'apprentissage des langues et l'ordre à suivre dans cet enseignement. La langue maternelle doit occuper la place la plus importante, renforcée par l'étude des éléments du slavon, la langue des livres saints et de la littérature ancienne. En second lieu viennent le français et l'allemand. A propos de la langue latine, Betzky remarque : «Il nous parait superflu d'enseigner aux cadets la langue latine. Cette langue sera efficacement suppléé par la langue française, aujourd'hui la plus généralement répandue et dans laquelle sont traduits tous les meilleurs auteurs anciens. » (Ibid., p. 58)

20 On y trouve également les conseils aux gouverneurs d'élèves qui doivent veiller « sur la conduite des enfants dès leur lever jusqu'à leur coucher» et contribuer au bon déroulement des études : "Végèce ${ }^{10}$ fournira encore de bonnes instructions pour les 
gouverneurs. On peut également apprendre de lui, l'art de guerre, et la langue française, par le moyen d'une bonne traduction, de l'édition de Paris de l'année 1759. Il sera utile aussi de profiter des Essaisde Montaigne dans les chapitres où il traite de l'éducation de la noblesse.» (Ibid., p. 48)

\section{Les emplois du temps}

21 Les Tableaux des Exercices et des Études de Messieurs les cadets des cinq classes (âges) rédigés pour l'année 1773 par le directeur des études Le Clerc apportent des détails sur l'organisation et le déroulement des cours.

Dans l'emploi du temps des cadets du second âge (de 9 à 12 ans), le russe, « la première des langues que les élèves russes doivent parler et écrire correctement ", aura huit heures de cours par semaine. Le Clerc souligne que « les maitres russes partageront le temps de leurs leçons entre la lecture et l'écriture. Ils s'attacheront principalement à la bonne prononciation ${ }^{11}$.

Le français occupera sept séances de deux heures chacune (lecture, écriture). A son sujet on trouve l'indication suivante: «Comme la langue française est après la russe celle qu'il importe le plus aux élèves de savoir à fond, on ne saurait trop la leur rendre familière, et comme elle est difficile, il faut les y exercer presque chaque jour et empêcher que dans les récréations et les repas on ne parle russe; les élèves n'oublieront jamais leur propre langue, mais ils peuvent oublier les autres ».

Notons que c'est l'inverse qui prévalait pour les élèves du premier âge (de 6 à 9 ans) : toute l'attention des préceptrices et des maîtresses ${ }^{12}$ devait être portée à la langue maternelle des élèves, au développement de l'expression orale et à l'apprentissage de la lecture et de l'écriture dans leur langue maternelle.

25 L'emploi du temps des cadets du second âge comporte d'autres matières. Ce sont les notions élémentaires d'arithmétique $(2 \mathrm{~h})$, les éléments d'une géographie simple qui soit à la portée des élèves et les productions des différents climats $(4 \mathrm{~h})$, la lecture du Dictionnaire d'Histoire naturelle et de Mythologie $(4 \mathrm{~h})$, le catéchisme $(1 \mathrm{~h})$.Elles sont enseignées dans la langue maternelle, qui est également utilisée pour les exercices de gymnastique, les jeux, la danse $(4 \mathrm{~h})$, le dessin $(4 \mathrm{~h})$.

Pour le troisième âge (de 12 à 15 ans), quatre heures sont réservées pour l'enseignement de la langue et de l'écriture russes. L'ouvrage précise : « ... sixmaîtres ${ }^{13}$ les enseigneront, lesélèves écriront sous la dictée; on corrigera soigneusement les fautes de prononciation et d'orthographe, en observant que ce n'est pas par nombre des pages qu'il faut compter, mais celui des phrases et des lignes, bien prononcées et bien écrites ".

Le français comporte dix heures hebdomadaires qui se partagent entre la grammaire, la dictée, la lecture ${ }^{14}$. Les consignes aux enseignants sont succinctement formulées : « ... on enseignera la langue française; on développera les principes de cette langue qui seront à la portée des élèves; ils écriront sous la dictée pendant une demi-heure; on leur dictera des choses agréables et instructives à la fois, également propres à orner l'esprit, et à inspirer l'amour des devoirs qui est le principe de toutes les vertus. On s'attachera à corriger la mauvaise prononciation, et les fautes d'orthographe. C'est de cette manière qu'on les exercera dans le style, qui doit être simple, correct et facile ».

28 Le français est progressivement introduit dans l'étude des autres matières. Pour le troisième âge(de 12 à 15 ans), on pratique pendant une heure, au cabinet d'histoire naturelle, la lecture des articles du Dictionnaire d'Histoire naturelle de Valmont de 
Baumare ${ }^{15}$, «... afin d'y puiser les connaissances que l'on désirera, sur les objets qui piqueront la curiosité ». On lit également et on explique la Fable [les dieux de l'Antiquité] ou la Mythologie, " afin que les élèves connaissent les sujets qu'elle a fournis à l'imitation des arts...».

Le Clerc signale à maintes reprises que les contenus et les modalités de l'enseignement dans toutes les matières doivent être à la portée des élèves et enseignés en conversant avec eux. Il souligne que «cette manière d'instruire et d'enseigner est préférable à celle qui donne les objets et les choses comme une tâche, un devoir à remplir ».

L'emploi du temps comprend aussi des cours d'allemand $(3 \mathrm{~h})$ qui sont consacrés à la lecture et à l'écriture dans cette langue.

31 Le quatrième âge (de 15 à 18 ans) et lecinquième âge(de 18 à 21 ans) ont six heures hebdomadaires de cours de russe qui comprennent la grammaire, l'orthographe, la langue esclavonne, la traduction du français ou de l'allemand en russe.

Dans l'emploi du temps, il n'y a plus d'heures qui soient uniquement réservées à l'étude de la langue française, mais plusieurs disciplines sont partiellement enseignées en français. En effet, pour les sciences et les disciplines militaires, Le Clerc évoque les ouvrages français sur lesquels les cadets de ces deux classes d'âge doivent se pencher avec application : l'Artillerie et la Tactique de Le Blond, l'Abrégé de Mécanique de Trabaut, l'Art de peindre à l'Esprit, la Logique de Le Clerc, un " excellent précis de Locke $~^{16}$, l'Histoire des voyagespar l'abbé Prévost, ou par une Compagnie de gens de Lettres anglais. On y trouve de nouveau le Dictionnaire de l'Histoire naturelle de Valmont de Baumare, l'Abrégé de l'Histoire des arts, etc.

33 Il est à noter que la composition de l'emploi du temps pour les élèves de tous les âges faisait partie des obligations de Le Clerc à l'époque où il occupait les fonctions de Directeur des Sciences.Mais selon le règlement du Corps, le directeur devait agir « ... de concert avec des inspecteurs» en ce qui concernait « les heures d'études, moyens d'instruction les plus faciles...», etc. (Ibid., p. 90)

\section{Principes et recommandations}

Les Tableaux des Exercices et des Études sont accompagnés de consignes et de recommandations aux maîtres et aux précepteurs qui rappellent les principes généraux de l'enseignement et de l'éducation dans l'établissement, par exemple :

Messieurs les Gouverneurs sont priés de se ressouvenir, que ce n'est pas la multiplicité des choses que l'on enseigne qui fait le prix et le mérite de l'éducation ; les connaissances doivent marcher par ordre; il faut exercer les sens avant que de parler à l'esprit, sans quoi on parlerait à un sourd: il ne faut donc pas que la tête des élèves ressemble à une bibliothèque renversée; il y a des tiroirs pour placer successivement toutes les connaissances. D'ailleurs, savoir peu, savoir bien, savoir des choses utiles, c'est savoir beaucoup; c'est là aussi le chef-d'œuvre de l'éducation. En suivant l'ordre méthodique de ce Tableau calqué sur la marche naturelle de l'esprit humain, Messieurs les Gouverneurs doivent bien étudier les dispositions, le penchant, le goût déterminé de Messieurs leurs élèves pour les arts, les sciences et les différents états de la société. L'aptitude, l'inclination étant bien connues, il faudra les diriger sagement vers le but que la nature aura marqué. On fait toujours bien ce que l'on fait avec plaisir, avec goût, avec amour; et au contraire, ce que l'on fait à regret et contre le vœu de la nature, on le fait toujours mal. Voilà pourquoi les enseignements uniformes ne produisent point les fruits qu'on en attend. [...] Les âmes bien conformées, ont un goût général pour tout ce qui est naturel, et en même temps un amour de préférence qui les attache à certains 
objets en particulier. C'est cet amour qui détermine, qui fixe les talents, et qui les conserve en les fixant. Il ne faut jamais perdre de vue cette observation importante. » (Premier Tableau)

l'enseignement des langues vivantes, qui, selon lui, doit être fondé, comme les autres
matières enseignées, sur les principes généraux de l'instruction et de l'éducation
prônés dans le projet de Betzky. dans une attestation qu'il donne en 1774 à Jean-Baptiste Maudru, l'un des maîtres de français de l'établissement qui avait mis en pratique une nouvelle méthode d'apprentissage de la lecture en français. Avant de venir en Russie Maudru avait longuement pratiqué sa méthode d'enseignement des langues vivantes, et il essaya de les diffuser par le biais de quelques publications faites en France et en Russie ${ }^{17}$. C'est en maître de langue expérimenté que Le Clerc donne une appréciation de l'enseignement de Maudru :

J'atteste que l'auteur du nouveau système de lecture applicable à toutes les langues, a fait usage de son système au Corps impérial des cadets nobles, à SaintPétersbourg, et que, selon cette nouvelle méthode, enseignée pendant un an, il est parvenu à faire lire très distinctement un grand nombre de cadets choisis, tous, parmi les moins avancés, et parmi ceux qui avaient le plus de difficulté à articuler les sons, à prononcer les mots, etc.; sans nullement les priver de leurs autres exercices journaliers et nombreux. À cet avantage, il s'en est joint plusieurs autres, tels, par exemple, que d'avoir détruit tout accent vicieux, d'avoir fait observer, en lisant, les règles de la ponctuation et de la prosodie, d'avoir mis à même ses élèves de déclamer avec grâce. Le même auteur leur a encore enseigné, par la même méthode, l'analyse grammaticale, l'analyse logique et l'analyse oratoire, toutes trois également bien et d'une manière sûre. C'est pourquoi, en considération de ce que cette méthode, applicable à toutes les langues, apprend à lire correctement avec la meilleure prononciation; fait connaître les vrais principes de la langue; peut former le cœur et l'esprit, par le choix des extraits qui entrent dans le plan de l'ouvrage: on l'a fait imprimer en partie, aux frais du Corps, pour l'usage des élèves; et l'on a accordé à l'auteur une gratification particulière de quatre cents roubles. À Saint-Pétersbourg, le $1^{\mathrm{er}}$ mai 1774, N. Clerc. méd. : de S.A.I., du Corps des Cadets, et directeur des études de ce Corps.

L'ouvrage de Maudru, imprimé aux frais du Corps ainsi que le mentionne l'attestation, fut intitulé l'Esquisse du nouveau système de lecture (1771). Il contenait une présentation de la méthode d'apprentissage des langues vivantes, des exercices de lecture et des extraits choisis des Aventures de Télémaque ${ }^{18}$. Le Clerc tenait à une organisation efficace de l'enseignement des langues, ce qui explique son intérêt pour les innovations de Maudru ainsi que le soutien qu'il apporta à l'auteur.

\section{Les autres établissements}

Le projet de Betzky s'étendait à d'autres établissements. L'Académie des Beaux-arts, l'Institution des jeunes filles Smolny, l'École de commerce inclurent également les 
langues vivantes étrangères dans leurs programmes d'études. Le but de l'apprentissage, fixé par les statuts de chaque établissement, était différent suivant les institutions, dans la mesure où il dépendait des objectifs spécifiques visés par chacune.

À Smolny, par exemple, l'étude du français par les jeunes filles ne jouait pas un rôle aussi fondamental qu'au Corps des cadets. Alors que les garçons suivaient un grand nombre de cours de grammaire, de lecture et de traduction, et que dans ces cours on les obligeait à communiquer avec leurs maîtres dans la langue étudiée, pour les filles de Smolny, ce fut plutôt l'inverse : l'expression orale et la communication dans la langue étudiée concernait essentiellement les sujets de la vie quotidienne qui eurent toujours chez elles plus d'importance que l'étude des difficultés et des particularités de la langue étrangère.

41 Dans la Russie du XVIII ${ }^{e}$ siècle, l'enseignement des langues vivantes ne se limitait pas aux établissements soumis aux projets de Betzky. Il fut dispensé durant tout le siècle par d'autres institutions fondées sur l'initiative de certains hommes d'État.

La première école qui adopta les langues étrangères dans ses programmes ouvrit à Moscou. Ce fut le gymnase du pasteur Ernst Glück, prisonnier de guerre, originaire de Saxe (1655-1705), qui maitrisait plusieurs langues anciennes et modernes y compris le russe et le slavon. L'établissement ne vécut que trois ans (1703-1706), au cours desquels Glück put cependant composer ou traduire quelques ouvrages pour ses élèves. Son enseignement s'inspirait entre autres des œuvres didactiques de Jan Amos Comenius ${ }^{19}$. Le français et l'allemand comptaient parmi les langues enseignées.

L'université de l'Académie des Sciences, fondée en 1724 à Saint-Pétersbourg, et l'université de Moscou (1755) intégraient en leur sein des gymnases qui devaient former de futurs étudiants pour ces deux premières universités de Russie : le gymnase académique (1724-1805) de Saint-Pétersbourg et les deux gymnases de l'université de Moscou (1756-1814). Ils avaient à peu près la même organisation et le même fonctionnement, inspirés des mêmes modèles occidentaux, surtout allemands. Le gymnase académique ne connut pas pourtant le lustre de ceux de l'université de Moscou.

44 Mixail Lomonossov (1711-1765), qui fut l'un des principaux acteurs des Lumières russes et l'un des protagonistes de ces institution ${ }^{20}$, comparait des universités dépourvues de gymnases à des champs labourés privés de semence. Il estimait que, pour former durablement une élite intellectuelle russe, la Russie devait favoriser le progrès de l'instruction pour des publics diversifiés.

45 Les deux gymnases ouverts auprès de l'université de Moscou, l'un pour les enfants de la noblesse et l'autre pour ceux de la bourgeoisie, appliquaient les principes inscrits dans le Règlement rédigé par Lomonossov; des filiales ouvrirent dans la ville de Kazan. Créés en même temps que l'université, ces gymnases devaient lui servir de pépinières. Johann Matthias Schaden (1731-1797), docteur en philosophie de l'université de Tübingen, fut invité en Russie sur l'initiative de Lomonossov pour devenir leur premier directeur (1756-1772). Partisan des doctrines philosophiques de Leibniz et de Christian Wolf, Schaden ne manifestait aucun enthousiasme pour le nouveau système pédagogique de Rousseau, ni pour les idées de Basedow qui furent plus tard en faveur dans les établissements de Betzky. Le programme d'études qu'il élabora pour les gymnases universitaires s'inspirait de conceptions assez traditionnelles, qu'il adaptait aux conditions de la société russe. En effet, ce programme réservait une place importante à 
l'étude des langues vivantes. Le français et l'allemand, qui étaient les grandes langues du savoir dans l'Europe des Lumières, étaient jugés indispensables au développement des jeunes sciences russes. Schaden divisa l'enseignement du gymnase en quatre cycles, ou « écoles » : russe, latine, des sciences fondamentales, des langues vivantes. Chaque « école » comportait trois «classes », à l'exception de l'école des langues vivantes qui en avait deux. L'étude des langues vivantes était obligatoire pour les élèves d'origine noble. On apprenait dans cette "école» les langues vivantes «les plus connues en Europe ", principalement le français et l'allemand, mais aussi l'anglais et même l'italien ${ }^{21}$.

Plus tard, s'ouvrit, auprès de l'université de Moscou, le Pensionnat des nobles (1776-1833), qui reproduisait en principe l'agencement des études adopté dans les gymnases universitaires. Mais pour l'organisation de la vie des pensionnaires,il était plutôt comparable aux établissements fermés de Betzky. Ce fut un établissement éducatif remarquable, très estimé par les contemporains, notamment pour l'enseignement des langues :

C'est selon la méthode et les règles bien élaborées et appréciées qu'on apprend à entendre et à parler les langues étrangères au Pensionnat de l'Université de Moscou. On y initie à traduire les pensées de l'auteur et non seulement ses mots, tout en préservant les beautés des langues, à rédiger avec la précision logique et la pureté rhétorique...22

Le programme pour le français et pour chaque langue étudiée projetait d'enseigner : 1) la lecture et l'écriture, 2) l'étymologie, 3) la syntaxe et la traduction, 4) la lecture des meilleurs auteurs et la rédaction de textes. Le programme de l'année 1790 donne une idée de l'organisation des cours de français :

... d) M. Matweï Brevnov, les quatre après-midi de $14 \mathrm{~h}$ à $18 \mathrm{~h}$ (lundi, mardi, jeudi, vendredi) apprendra à lire et à écrire en français ; pour que les élèves s'habituent dès le début à bien prononcer et à communiquer facilement dans cette langue, il les incitera de surcroît à s'exprimer en français sur des sujets différents et à réciter des petits textes traduits du russe en français.

Le mercredi et le samedi, de $14 \mathrm{~h}$ à $16 \mathrm{~h}$, il proposera à ses élèves, pour le meilleur progrès en langue française, les différents exercices et questions tirés des ouvrages de géographie et de l'abrégé de toutes les sciences.

e) Charles Aviat de Vatay enseignera les règles de la grammaire française les quatre après-midi de $14 \mathrm{~h}$ à $18 \mathrm{~h}$. En première classe, il consacrera son temps à l'écriture, à l'explication des règles de grammaire et à la lecture des auteurs étudiés en classe. Le mardi, en lisant les meilleurs auteurs français, il interprétera leurs pensées tout en montrant la pureté du style. Il fera faire des traductions qu'il corrigera. Pour les leçons, il préparerales expressions et les tours de langue les plus employés, il encouragera ses élèves à exprimer leurs pensées en français par écrit, et de vive voix encore davantage, pour qu'il puissent progresser dans l'étude. $»^{23}$

Notons que l'enseignement dans les gymnases faisait souvent partie des charges des professeurs des universités. Ainsi, Charles Aviat de Vatay (17??-1809), qui enseignait au pensionnat, était en même temps professeur en langue et lettres françaises à l'université de Moscou. Il traduisit plusieurs écrivains russes en français et rédigea un cours d'histoire de la littérature française à l'usage de ses étudiants.

Dans les deux capitales, fonctionnèrent aussi des gymnases de communautés étrangères. Une école allemande (Peterschule) de la communauté luthérienne regroupée autour de l'église Saint Pierre (1783-1917) bénéficiait d'une excellente réputation à Saint-Pétersbourg. C'est là qu'un disciple de Basedow, Christian Wolke (1741-1825), qui avait été invité en Russie par Catherine II, lança dans les années 1780 sa 
méthode d'enseignement des langues vivantes. L'enseignement du français eut de solides fondements dans le gymnase pour les étrangers chrétiens orthodoxes (1769-1796) à Saint-Pétersbourg. Dès 1798, le français et l'allemand s'ajoutèrent à l'étude du grec ancien, du latin et de l'hébreu dans les quatre " académies spirituelles " de Kiev, de Moscou, de Saint-Pétersbourg et de Kazan, qui formaient le haut clergé orthodoxe.

Dans plusieurs villes furent créés des « pensionnats ", établissements éducatifs privés, tenus généralement par des étrangers, Français, Allemands ou Suisses. Leur nombre augmenta surtout vers la fin du XVIII ${ }^{\mathrm{e}}$ siècle, quand la Russie donna refuge aux aristocrates et aux ecclésiastiques français qui fuyaient la Révolution. Certains d'entre eux acquirent un véritable renom: ceux de l'abbé Nicolle, de Ferrat, de Modérach. L'abbé Dominique-Charles Nicolle (1758-1835) accompagna dans l'émigration en Russie son protecteur le duc de Richelieu (1793). Il était entouré de l'équipe pédagogique du collège parisien Sainte Barbe dont il avait été le directeur. Le pensionnat qu'il organisa à Saint-Pétersbourg (1796-1809) offrait ses prestations aux familles les plus riches de la noblesse russe auxquelles il assurait une instruction et une éducation solides. Tout l'enseignement y était donné en français.

51 Le développement et l'augmentation du nombre des établissements éducatifs n'entraînèrent pas immédiatement la disparition de l'éducation et de l'enseignement donnés dans les familles. La noblesse russe continuait à confier ses enfants aux précepteurs étrangers auxquels elle avait eu recours depuis les années 1730. Mais dans la seconde moitié du siècle, les exigences vis-à-vis de la qualité des prestations éducatives évoluèrent rapidement. Dès 1757 , pour exercer leur métier, les précepteurs étrangers devaient obtenir une autorisation auprès d'une commission universitaire qui confirmait leurs compétences. Dans les dernières années du siècle, la connaissance de la langue des élèves devint pour eux obligatoire.

L'apprentissage du français et des autres langues vivantes fut étroitement lié aux établissements mentionnés précédemment qui apparurent et se développèrent en Russie à partir du milieu du XVIII siècle. C'est avec la création des établissements d'éducation au milieu du XVIII ${ }^{e}$ siècle que débuta la publication d'ouvrages didactiques, qui ne cessa de s'amplifier et de se diversifier. Les auteurs ou les adaptateurs de ces ouvrages à l'usage des russophones les destinaient avant tout aux élèves des établissements pour lesquels ils travaillaient. Plusieurs institutions où les langues vivantes furent enseignées possédaient leurs propres imprimeries: l'université de Moscou, l'Académie des Sciences, le Corps des cadets, le Corps de la Marine. De nombreuses imprimeries privées vinrent également à leur secours dans les années 1770 et 1780 . Le système éducatif russe qui traversa plusieurs réformes durant la période suivante (XIXe-début du XXe siècle) prit soin de l'enseignement des langues vivantes et du renouvellement de ses outils didactiques.

54 Aujourd'hui, la nombreuse documentation relative à l'enseignement des langues au $\mathrm{XVIII}^{\mathrm{e}}$ et au XIX ${ }^{\mathrm{e}}$ siècle dans la Russie des tsars est éparpillée dans les fonds des bibliothèques nationales et régionales. Après la révolution de 1917 qui abolit l'ancien système éducatif, ces bibliothèques ont accueilli et protégé des collections de livres, entières ou partielles, qui avaient appartenu aux institutions éducatives de la Russie impériale. Ainsi, la collection des livres du Corps des cadets, l'une des plus prestigieuses, se trouve à Saint-Pétersbourg dans les réserves de la Bibliothèque 
nationale et de celle de l'Académie des Sciences. Elle est en cours de regroupement et sera bientôt accessible aux chercheurs. La renaissance de plusieurs établissements éducatifs, le fait que la Russie postsoviétique s'attache à renouer avec les traditions des siècles précédents favorisent l'étude de l'héritage culturel grâce au développement des recherches historiques qui se sont multipliées rapidement au cours des dernières années. Par conséquent, il devient désormais possible de reconstituer les corpus d'ouvrages didactiques qui ont été en usage dans chaque type d'établissement. Leur étude permettra de connaître et d'évaluer l'enseignement du français tel qu'il a été donné en Russie au XVIII et au XIX siècle, sur la base des documents authentiques, qui sont encore le plus souvent inconnus des historiens de la didactique des langues.

\section{NOTES}

1. Les langues vivantes (l'allemand, le français, l'anglais) furent intégrées dans les programmes de toutes les écoles militaires formant les officiers dès leur fondation : le Corps des cadets de la Marine (1715), le Corps des cadets de l'Artillerie et du Génie (1719) et le Corps des cadets nobles (1731), mais les deux premiers établissements ne furent pas inclus dans le projet de Betzky.

2. Plus tard, en 1786, les desseins de Catherine dans le domaine de l'éducation prirent une dimension plus large et elle envisagea la création d'établissements d'enseignement public dans tout l'empire. Les centres administratifs de plusieurs provinces devaient ouvrir des écoles de deux types (petites et principales) donnant accès aux études à des enfants de diverses origines sociales. L'élaboration et la mise en place de ce projet grandiose furent l'œuvre d'un autre personnage important de l'époque, Théodore Yankovič de Mirievo (1741-1814), pédagogue d'origine serbe, nourri des idées pédagogiques de Jan Amos Comenius et des innovations de Iohann Ignaz Felbiger (1724-1788). Ayant été longtemps au service de la cour autrichienne, Yankovič réforma avec succès le système de l'éducation publique du pays pendant le règne de Marie-Thérèse qui lui offrit un titre de noblesse pour ce service. En 1782, Yankovič, appelé désormais Yankovič de Mirievo (son lieu de naissance près de Belgrade), vint en Russie sur l'invitation de Catherine et avec une recommandation de Joseph II afin d'y accomplir la même tâche, d'organiser et de faire fonctionner le système de l'enseignement public dans tout l'empire russe.

Les programmes d'études créés par Betzky, et plus tard par Yankovič de Mirievo à l'usage d'établissements très différents du point de vue de la finalité des études aussi bien que des publics, incluaient les langues vivantes en tant que disciplines obligatoires, quoique leur apprentissage n'y fût pas d'importance égale.

Dans les écoles créées par Yankovič de Mirievo et destinées aux enfants de tous les milieux sociaux (à l'exception de ceux des serfs) la place des langues vivantes était bien modeste, leur apprentissage ne débutait qu'avec le passage des élèves d'une petite école à la principale. Le choix d'une langue vivante dépendait des besoins pratiques de la région où l'école ouvrait. Par exemple, l'étude du chinois était prévue dans les régions avoisinant la Chine.

3. Ivan Betzky avait élaboré son projet concernant l'instruction et l'éducation de la jeune élite russe apparemment bien avant la commande de l'impératrice, pendant son séjour prolongé à Paris entre 1756 et 1762. Voir : N. G. Le Clerc, 1775, Introduction du traducteur, note (a), p. 4 (cf. ci-dessous, note 7 pour la référence complète de l'ouvrage) ; Russkij biografičeskij slovar' izdavaemyj 
Russkim Istoričeskim Obščestvom, Sankt-Peterburg, 1914, p. 6-7 [Dictionnaire biographique russe édité par la Société Historique de Russie, Saint-Pétersbourg, 1914, p. 6-7].

4. Par ailleurs, Catherine II en fut très fière. A ce sujet elle écrivit : «On a beau croire [...] que mes Cadets sont formés uniquement pour la guerre... Mes Cadets sauront devenir ce qu'ils voudront, en choisissant un domaine d'activité selon leurs goûts et leurs facultés ». Cité dans : P. N. Milukov, Očerki po istorii russkoj kul'tury, t. 2. Moskva, 1994, p. 267 [P. N. Milukov, Essais sur l'histoire de la culture russe, t. 2, Moscou, 1994, p. 267].

5. C'est seulement depuis une dizaine d'années que l'on voit émerger l'intérêt des chercheurs russes pour l'étude de la réception des idées philosophiques et pédagogiques des Lumières, ainsi que pour le fonctionnement des divers établissements éducatifs dans la Russie des XVIII ${ }^{\mathrm{e}}$-XIX ${ }^{\mathrm{e}}$ siècles. Voir, par exemple : V. Blinov, Razvitie teorii i praktiki obrazovanija $v$ Rossii XVIII-načala XX veka pod vlijaniem cennostnyx orientacij, predstavlenij ob ideale čeloveka $v$ celjax ego vospitanija, Moskva, 2003 [L'influence des concepts pédagogiques de l'idéal de l'homme et des valeurs humaines sur la théorie et les pratiques de l'éducation dans la Russie du XVIII ${ }^{\mathrm{e}}$ jusqu'au début du $\mathrm{XX}^{\mathrm{e}}$ siècle, Moscou, 2003] ; S. Ja. Karp, «Le point de vue des physiocrates suédois sur les établissements d'enseignement de Catherine II (1773-1775) ", Cahiers du Monde russe, 43/2-3, 2002, p. 323-344 ; A. I. Lubžin, Očerki po istorii rossijskogo obrazovanija imperatorskoj epoxi, Moskva, 2000 [Essais sur l'histoire de l'instruction et de l'éducation en Russie impériale, Moscou, 2000], etc. Cette nouvelle interrogation du passé est due aux besoins pratiques de l'éducation nationale actuelle qui renoue avec la tradition d'avant la révolution de 1917, en explorant son héritage pédagogique. Le système de l'Éducation nationale en Russie est en train de changer. Des gymnases, des lycées, des Corps des cadets sont de retour dans le paysage éducatif, coexistant avec l'école secondaire générale, œuvre de la période soviétique.

6. Les compétences de directeur des études furent ainsi définies par les Statuts du Corps : «Le Directeur, choisi entre les hommes savants, sera versé dans toutes les sciences militaires et civiles, et doit joindre à toutes ces connaissances un mérite distingué et toutes les qualités propres à remplir une place aussi importante »(Le Clerc, 1775, t. 2, p. 90. Pour le titre détaillé, voir la note suivante).

7. Les Plans et les Statuts des différents Etablissements ordonnés par Sa Majesté Impériale Catherine II pour l'Education de la Jeunesse et l'Utilité générale de son Empire, Ecrits en Langue Russe, par Mr. Betzky et traduits en Langue Française, d'après les Originaux, par M. Clerc,Amsterdam, Marc-Michel Rey, 1775, 2 tomes. Le Clerc a également traduit du russe en français le texte de Betzky Éducation morale et physique des deux sexes pour les rendre aussi utiles aux autres qu'à eux-mêmes (Besançon, 1777), ainsi que plusieurs œuvres littéraires, entre autres des poèmes de Lomonossov et de Khéraskov, et une pièce de théâtre de Catherine II.

8. Voir à ce sujet Georges Dulač, "Diderot, éditeur des Plans et statuts des établissements de Catherine II ", Dix-huitième siècle, 16, 1984, p. 323-344.

9. C'est-à-dire le slavon, langue liturgique et savante.

10. Végèce, en latin Flavius Vegetius Renatus, fin du IV e siècle apr. J.-C., l'auteur d'un Traité de l'Art militaire.

11. Il y a une remarque de Le Clerc qui met en valeur l'attention particulière portée au maintien chez les élèves d'un bon niveau dans leurs langues maternelles autres que le russe : "Quant aux Livoniens, la langue allemande qui est leur langue naturelle, ne doit pas être négligée; il faut donc les exercer dans cette langue au moins deux fois la semaine; et s'il se trouvait parmi Messieurs les cadets du second âge quelques élèves qui voulussent l'apprendre avec eux, on ne négligera rien pour féconder leur bonne volonté» (t. 2, Premier Tableau).

12. Les cadets du premier âge étaient encadrés par un personnel féminin.

13. Chaque groupe est composé de vingt à vingt-cinq élèves.

14. Les leçons d'écriture française sont évidemment considérées comme moins importantes à cette étape, étant assurées seulement par deux maîtres; soixante élèves en même temps pour chacun des deux maîtres. 
15. Jacques-Christophe Valmont de Baumare (1731-1807), naturaliste, auteur du Dictionnaire raisonné d'Histoire naturelle. Ce Dictionnaire était déjà familier aux élèves car dans l'âge précédent ils en avaient vu « les articles les plus importants » (t. 2, Premier Tableau).

16. Il s'agit de l'Essai philosophique concernant l'entendement humain, où l'on montre quelle est l'étendue de nos connaissances certaines, et de manière dont nous y parvenons. Traduit de l'Anglais par M. Coste. Amsterdam, 1742 (4 édition). Ce traité de Locke était de même connu en Russie sous forme de l' Abrégé de l'Essai... sur l'entendement humain, Londres, 1746, effectué par Jean Le Clerc.

17. Jean-Baptiste Maudru (17 ??-18 ??) est plus connu comme auteur de la première grammaire du russe publiée en France: Eléments raisonnés de la langue russe ou principes généraux de la grammaire appliqués à la langue russe, Paris, 1802. Les approches de l'enseignement des langues de cet auteur transparaissent de ses publications précédant la publication de sa grammaire russe. En effet, l'aperçu de son programme linguistique et didactique ayant pour titre L'esquisse du nouveau système de lecture ou Lettre à Madame la Duchesse de *** fut publié pour la première fois par son auteur à Paris en 1771. Il les exposa ensuite dans une adaptation didactique du Télémaque de Fénelon, publiée sous le titre de Prospectus(Saint-Pétersbourg, 1776) à l'usage des russophones, ainsi que dans ses livres élémentaires de lecture. Plus tard il publia ses Réflexions sur l'éducation d'abord à Saint-Pétersbourg (1778) et ensuite à Paris (1792). La synthèse de ses idées se trouve dans son ouvrage capital Le nouveau système de lecture applicable à toutes les langues, Paris, 1800.

18. Il est intéressant de constater que ce système de lecture intéressa plus tard Yancovič de Mirievo qui y eut éventuellement recours en élaborant ses manuels élémentaires de lecture russe. Dans l'Avant-propos de ses Réflexions... (1792) Maudru dit que Jankovič de Mirievo, intéressé par son système de lecture, lui avait demandé de le lui prêter pour en faire une copie.

19. Il s'agit notamment de la Janua linguarum reserata (1631) et de Orbis sensualium pictus (1658).

20. M. Lomonossov rédigea le Règlement pour les deux gymnases auprès de l'université de Moscou. Il assura la direction du gymnase académique à Saint-Pétersbourg entre 1758 et 1765 et y imposa plusieurs transformations.

21. Il est intéressant de noter que, dans le règlement pour le gymnase d'enfants de roturiers (1753), Lomonossov souligna une nécessité d'apprendre les langues vivantes aussi à cette catégorie d'élèves: "Au passage des élèves du gymnase en catégorie "étudiants de l'université" il faut tenir compte de leur âge. Ceux qui ont réussi vers quinze ans à faire dans toutes les classes du russe, du latin et des sciences fondamentales, devront suivre le même enseignement du français et de l'allemand qui est dispensé aux élèves du gymnase des nobles. »

22. M. T. Kačenovskij, «Vzgljad na Blagorodnyj pansion pri Imperatorskom Moskovskom Universitete », Vestnik Evropy, 1804, 19, p. 223 ["Un regard porté sur le Pensionnat des Nobles auprès de l'Université Impériale à Moscou », Le Messager de l'Europe, 1804, 19, p. 223]

23. Kratkoe načertanie učebnyx klassov $i$ voobšče vsego porjadka, nabljudaemogo pri soderžanii Vol'nogo Blagorodnogo Pansiona... Moskva, V. Universitetskoj tipografii, u V. Okorokova, 1790 [Abrégé du programme d'études et du règlement du Libre Pensionnat des Nobles auprès de l'Université Impériale de Moscou, Moscou, Imprimerie de l'Université, V. Okorokov, 1790, 16 p.]

\section{RÉSUMÉS}

Le but de cet article est de soulever un coin du voile sur l'enseignement du français et des autres langues vivantes dans la Russie impériale du XVIII ${ }^{\mathrm{e}}$ siècle, époque où elle construisait son 
système éducatif en puisant dans les idées et l'expérience des pays occidentaux et en leur faisant épouser les conditions russes. La réalisation du projet éducatif de Betzky pour plusieurs établissements fermés, en particulier le déroulement de l'apprentissage des langues dans le Corps des cadets, ainsi que l'organisation des cours de langues dans les gymnases universitaires et les pensionnats sont décrits à travers les témoignages des acteurs et des documents de l'époque inédits ou peu connus dans l'histoire de la didactique des langues.

The aim of this article is to uncover some aspects of the teaching of French and other modern languages in $18^{\text {th }}$ century Russia, when it was building its education system by drawing from the ideas and experience of western countries and by adapting them to Russian conditions. The way Betsky's educational project was achieved in several boarding schools, and in particular the language curriculum designed for the Cadet Corps, as well as the language classes format used in prep schools and private schools are described from witnesses and unpublished or little known contemporary documents.

\section{INDEX}

Mots-clés : enseignement du français, Russie impériale, projet éducatif, établissements fermés, cours de langue

Keywords : teaching of French, imperial Russia, educational project, boarding schools, language classes

\section{AUTEUR}

\section{NADEJDA KOUZMINA}

Université Marc Bloch, Strasbourg 